\title{
A FEASIBILITY STUDY ON USING ViSP'S 3D MODEL-BASED TRACKER FOR UAV POSE ESTIMATION IN OUTDOOR ENVIRONMENTS
}

\author{
Julien Li-Chee-Ming and Costas Armenakis \\ Geomatics Engineering, GeoICT Lab \\ Department of Earth and Space Science and Engineering \\ Lassonde School of Engineering, York University \\ 4700 Keele Str., Toronto, Ontario, M3J 1P3 \\ \{julienli\}, \{armenc\} @yorku.ca
}

Commission I, ICWG I/Vb

KEYWORDS: UAV, Close Range Photogrammetry, Mapping, Tracking, Robotics, Navigation, Object Recognition, Augmented Reality, ViSP

\begin{abstract}
:
This paper presents a novel application of the Visual Servoing Platform's (ViSP) for small UAV pose estimation in outdoor environments. Given an initial approximation for the camera position and orientation, or camera pose, ViSP automatically establishes and continuously tracks corresponding features between an image sequence and a 3D wireframe model of the environment. As ViSP has been demonstrated to perform well in small and cluttered indoor environments, this paper explores the application of ViSP for UAV mapping of outdoor landscapes and tracking of large objects (i.e. building models). Our presented experiments demonstrate the data obtainable by the UAV, assess ViSP's data processing strategies, and evaluate the performance of the tracker.
\end{abstract}

\section{INTRODUCTION}

Precise navigation is required for UAVs operating in GPS denied environments, or in dense-multipath environments such as urban canyons. This is especially useful in urban missions, where the possibility of crashing is high, as UAVs fly at low altitudes among buildings and in strong winds, whiles avoiding obstacles and performing sharp maneuvers. The GeoICT Lab at York University is developing mapping and tracking systems based on small unmanned aerial systems such as the Aeryon Scout quadrotor small UAV (Unmanned Aerial Vehicle) (Aeryon, 2015). The Scout is equipped with a single frequency GPS sensor that provides positioning accuracies of about $3 \mathrm{~m}$, and an Attitude and Heading Reference System (AHRS) that estimates attitude to about $3^{\circ}$. It is also equipped with a small forward-looking FPV (First Person Viewing) video camera and a transmitter to downlink the video signal wirelessly in realtime to a monitoring ground station or to virtual reality goggles. FPV gives the operator of a radio-controlled UAV a perspective view from the 'cockpit'. This augments the visual line-of-sight, offering additional situation awareness. FPV systems are used solely as a visual aid in remotely piloting the aircraft.

We have proposed a method to further extend the application of this system by estimating the pose (i.e. position and orientation) of the UAV from the FPV video as it travels through a known 3D environment. We have applied a geometric hashing based method to match the extracted linear features from the FPV video images with a database of vertical line features extracted from synthetic images of the 3D building models (Li-Chee Ming and Armenakis, 2014). Sub-meter positional accuracies in the object space were achieved when proper viewing geometric configuration, control points, and tie points are used. The obtained quantitative information on position and orientation of the aerial platform supports the UAV operator in navigation and path planning. If an autopilot is available, this system may also be used to improve the navigation solution's position and orientation.

\section{VISUAL SERVOING AND VISUAL TRACKING}

In this paper we extend on our previous work where a 3D CAD model of the environment is available. We assess the Visual Servoing Platform's (ViSP) registration techniques which provide continuous tracking and alignment of features extracted from the FPV video image (in real-time) and the 3D model of the environment (Marchand and Chaumette, 2005). ViSP provides tracking techniques that are divided into two classes: feature-based and model-based tracking. The first approach focuses on tracking 2D geometric primitives in the image, such as points (Shi and Tomasi, 1994), straight line segments (Smith et al., 2006), circles or ellipses (Vincze, 2001), or object contours (Blake and Isard, 1998). However, ViSP does not provide a means to match these tracked image features with the 3D model, which is required in the georeferencing process. The second method is more suitable because it explicitly uses a $3 \mathrm{D}$ model of the object in the tracking algorithm. Further, a byproduct of this tracking method is the 3D camera pose, which is our main objective. This second class of methods usually provides a more robust solution (for example, it handles partial occlusion of the objects, and shadows).

ViSP has demonstrated its capabilities in applications such as augmented reality, visual servoing, medical imaging, and industrial applications (ViSP, 2013). These demonstrations involved terrestrial robots and robotic arms, equipped with cameras, to recognize and manipulate small objects (e.g. boxes, tools, and cups) in cluttered indoor environments. This paper explores the application of ViSP in mapping large outdoor environments by UAVs, and tracking larger objects (i.e. building models). Our presented experiments demonstrate the data obtainable by the UAV, assess ViSP's data processing strategies, and evaluate the performance of the tracker. The overall workflow is shown in Figure 1. Firstly, a Moving Edges Tracker identifies corresponding features between an image sequence and a 3D model. The pose is fed back into the Moving Edges Tracker. The following sections explain the algorithms in more detail. 


\subsection{The Moving Edges Tracker}

The moving edges algorithm (Boutemy, 1989) matches image edges in the video image frames to the 3D model's edges, which are called model contours in ViSP. The required initial approximation of the camera pose is provided by the UAV's autopilot GPS positioning, and orientation from the AHRS. Observations from these sensors are no longer used after initializing the camera pose in order to assess ViSP's tracking performance.

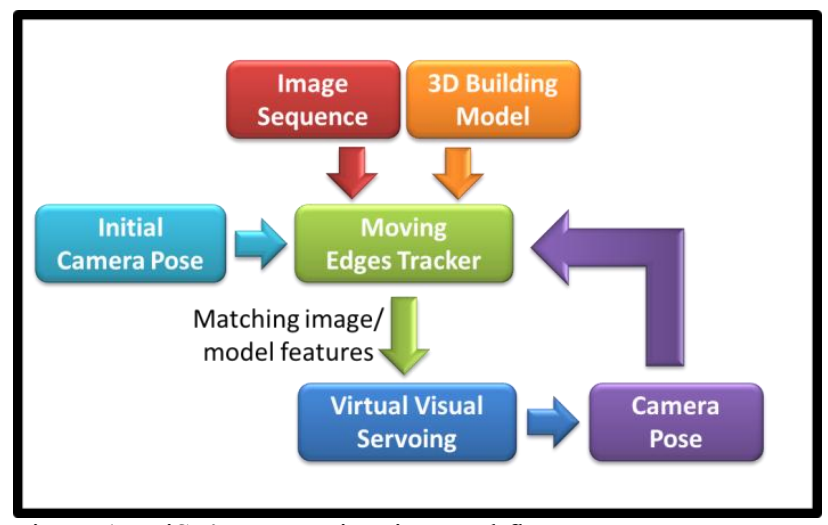

Figure 1. ViSP's pose estimation workflow.
The process consists of searching for the corresponding point $\mathrm{p}^{\mathrm{t}+1}$ in the image $\mathrm{I}^{\mathrm{t}+1}$ for each point $\mathrm{p}^{\mathrm{t}}$. A 1D search interval $\left\{\mathrm{Q}_{\mathrm{i}}\right.$, $\mathrm{j} \in[-\mathrm{J}, \mathrm{J}]\}$ is determined in the direction $\delta$ of the normal to the contour. For each position $\mathrm{Q}_{i}$ lying the direction $\delta$, a mask convolution $\mathrm{M}_{\delta}$ corresponding to the square root of a loglikelihood ratio $\zeta_{\mathrm{i}}$ is computed as a similarity measure. Thus the new position $\mathrm{p}^{\mathrm{t}+1}$ is given by:

$$
Q^{j *}=\operatorname{argmax}_{j \in[-J, J]} \zeta_{j}
$$

with

$$
\zeta_{j}=\left|I_{v\left(Q_{j}\right)}^{t+1} * M_{\delta}+I_{v\left(p^{t}\right)}^{t} * M_{\delta}\right|
$$

$v($.$) is the neighbourhood of the considered pixel, ViSP's$ default is a 7x7 pixel mask (Comport et al., 2003).
A)

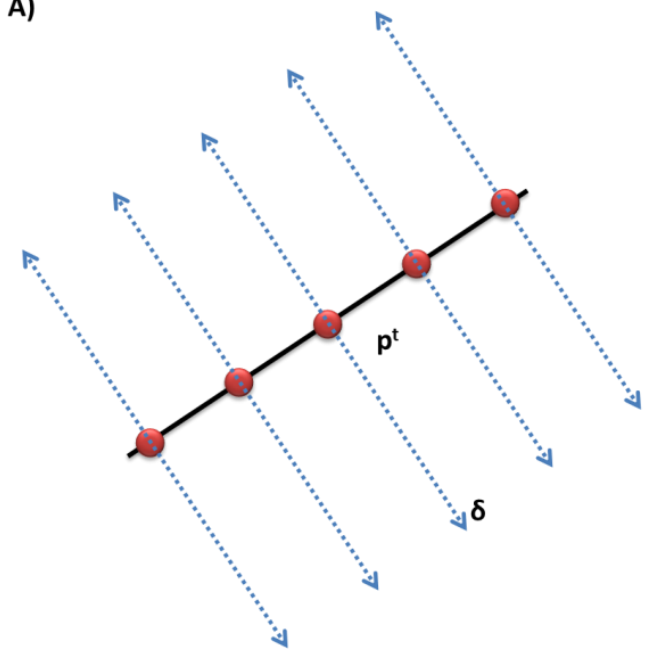

B)

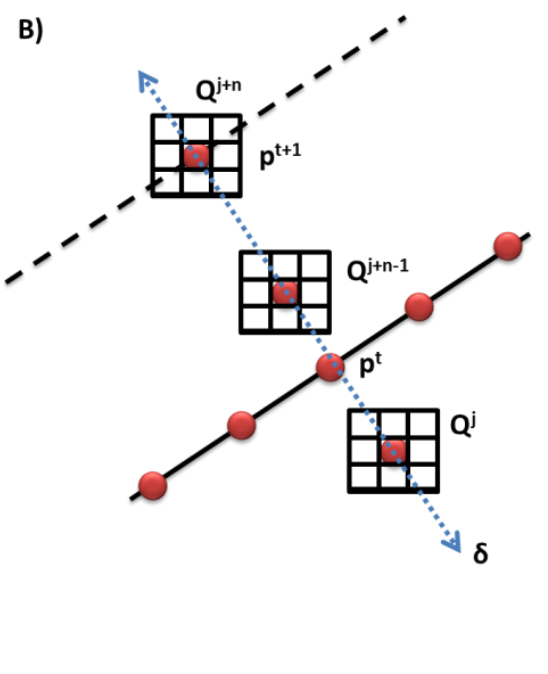

C)

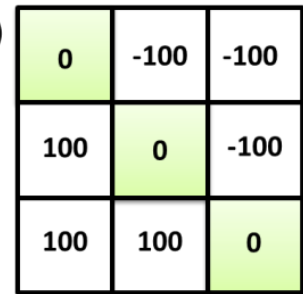

D)

\begin{tabular}{|c|c|c|}
\hline 100 & 100 & 100 \\
\hline 0 & 0 & 0 \\
\hline-100 & -100 & -100 \\
\hline
\end{tabular}

Figure 2. Determining point position in the next image using the oriented gradient algorithm: A) calculating the normal at sample points, B) Sampling along the normal, C)-D) 2 out of the $1803 \times 3$ predetermined masks, C) $180^{\circ}$, D) $45^{\circ}$ (Comport et al., 2003).

Model contours are sampled at a user specified distance interval (Figure 2A). At these sample points (e.g. pt), a one dimensional search is performed along the normal direction $(\delta)$ of the contour for corresponding image edges (Figure 2B). An oriented gradient mask is used to detect edges (e.g. Figures $2 \mathrm{C}$ and 2D). One of the advantages of this method is that it only searches for image edges which are oriented in the same direction as the model contour. An array of 180 masks is generated off-line which is indexed according to the contour angle. The run-time is limited only by the efficiency of the convolution, which leads to real-time performance (Comport et al., 2003). Line segments are favourable features to track because the choice of the convolution mask is simply made using the slope of the contour line. There are trade-offs to be made between real-time performance and both mask size and search distance.

\subsection{Virtual Visual Servoing}

ViSP treats pose estimation as a $2 \mathrm{D}$ visual servoing problem as proposed in (Sunareswaran and Behringer, 1998). Once each point's search along its normal vector finds a matching model point via the moving edges tracker, the distance between two corresponding points is minimized using a non-linear optimization technique called Virtual Visual Servoing (VVS). A control law adjusts a virtual camera's pose to minimize the distances, which are considered as the errors, between the observed data $s_{d}$ (i.e. the positions of a set of features in the image) and $s(\mathbf{r})$, the positions of the same features computed by forward-projection of the $3 \mathrm{D}$ features $\mathbf{P}$. For instance in the misclosure Equation (3), ${ }^{\mathbf{0}} \mathbf{P}$ are the $3 \mathrm{D}$ coordinates of the model's points in the object frame, according to the current extrinsic and intrinsic camera parameters: 


$$
\Delta=\left(s(\boldsymbol{r})-s_{d}\right)=\left[p r_{\xi}\left(\boldsymbol{r},{ }^{o} \boldsymbol{P}\right)-s_{d}\right]
$$

where $\operatorname{pr}_{\xi}\left(\boldsymbol{r},{ }^{o} \boldsymbol{P}\right)$ is the projection model according to the intrinsic parameters $\xi$ and camera pose $\mathbf{r}$, expressed in the object frame. It is assumed the intrinsic parameters are available, but VVS can estimate them along with the extrinsic parameters. An iteratively re-weighted least squares (IRLS) implementation of the M-estimator is used to minimize the error of the summation $\Delta$ squares. IRLS was chosen over other Mestimators because it is capable of statistically rejecting outliers.

Comport et al. (2003) provide the derivation of ViSP's control law. If the corresponding features are well chosen, there is only one camera pose that allows the minimization to be achieved. Conversely, convergence may not be obtained if the error is too large.

\subsection{TIN to Polygon 3D Model}

ViSP specifies that a 3D model of the object to track should be represented using VRML (Virtual Reality Modeling Language). The model needs to respect two conditions:

1) The faces of the modelled object have to be oriented so that their normal goes out of the object. The tracker uses the normal to determine if a face is visible.

2) The faces of the model are not systematically modelled by triangles. The lines that appear in the model must match image edges.

Due to the second condition, the 3D building models used in the experiments had to be converted from TIN (Triangulated Irregular Network) to 3D polygon models. The algorithm developed to solve this problem is as follows:

1) Region growing that groups connected triangles with parallel normals.

2) Extract the outline of each group to use as the new polygon faces.

The region growing algorithm was implemented as a recursive function. A seed triangle (selected arbitrarily from the TIN model) searches for its neighbouring triangles, that is, triangles that share a side with it, and have parallel normals. The neighbouring triangles are added to the seed's group. Then each neighbour looks for its own neighbours. The function terminates if all the neighbours have been visited or a side does not have a neighbour. For example, the blue triangles in Figure 3 belong to one group.

Once all of the triangles have been grouped, the outline of each group is determined (the black line in Figure 3). Firstly, all of edges that belong to only one triangle are identified, these are the outlining edges. These unshared edges are then ordered so the end of one edge connects to the start of another. The first edge is chosen arbitrarily.

Figure 4A shows the 3D TIN model of York University's Lassonde Buillding. Figure 4B shows the resulting polygon model of the same building.

\section{RESULTS AND DISCUSSION}

The 3D virtual building model of York University's Keele Campus campus (Armenakis and Sohn, 2009) was used as a known environment. The model consists of photorealistic 3D TIN reconstructions of buildings, trees, and terrain (Figure 5). The models were TIN (Triangulated Irregular Network) reconstructions of buildings, trees, and terrain. The model was generated from building footprint vector data, Digital Surface Model (DSM) with $0.75 \mathrm{~m}$ ground spacing, corresponding orthophotos at $0.15 \mathrm{~m}$ spatial resolution and terrestrial images. The 3D building models were further refined with airborne lidar data having a point density of 1.9 points per square metre (Corral-Soto et al., 2012).

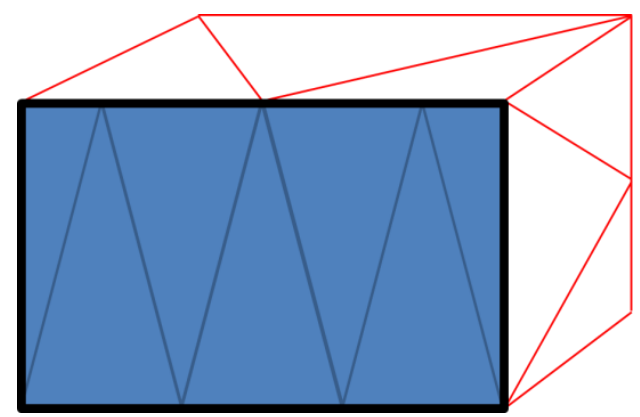

Figure 3. An example of region growing and outline detection: The blue triangles belong to a group because one triangle is connected to at least one other triangle with a parallel normal. The outline of the group (black line) consists of the edges that belong only to one triangle.
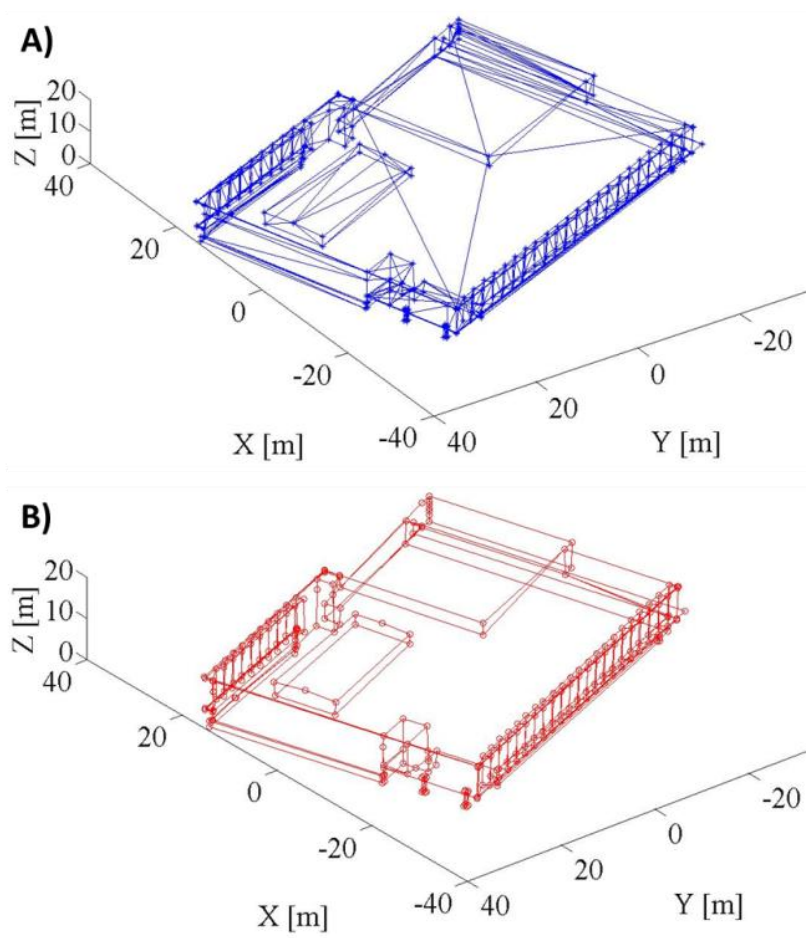

Figure 4. Converting TIN building models to polygon models. A) The original TIN model of the York University's Lassonde Building. B) The resulting 3D polygon model of the Lassonde Building. 
The 3D CAD model serves two purposes in the proposed approach. Firstly, it provides the necessary level of detail such that individual buildings can be uniquely identified via ViSP's moving edges algorithm. Secondly, it provides ground control points to photogrammetrically estimate the camera pose. The geometric accuracy of the building models is in the order of 10 to $40 \mathrm{~cm}$.

In our tests we used the data of an Aeryon Scout quadcopter which flew over York University, up to approximately 40 metres above the ground, while its onboard camera focused on buildings, walkways, and trees. Figure 6 shows the UAV's flight path for the presented experiment.

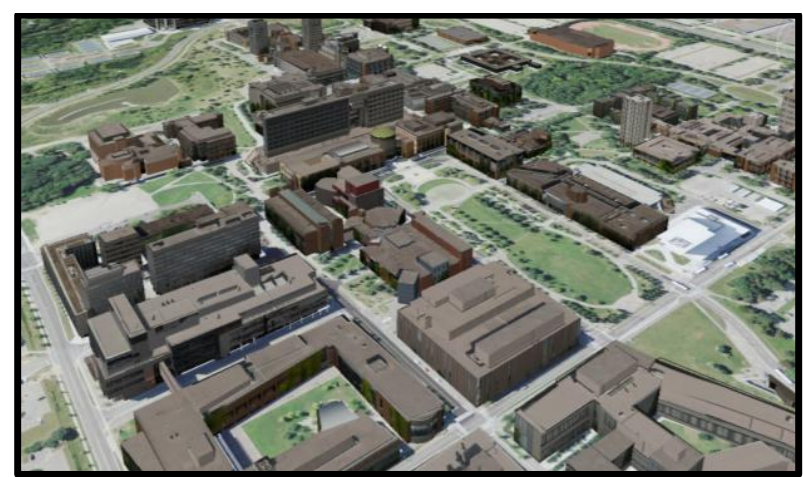

Figure 5. York University's 3D campus model

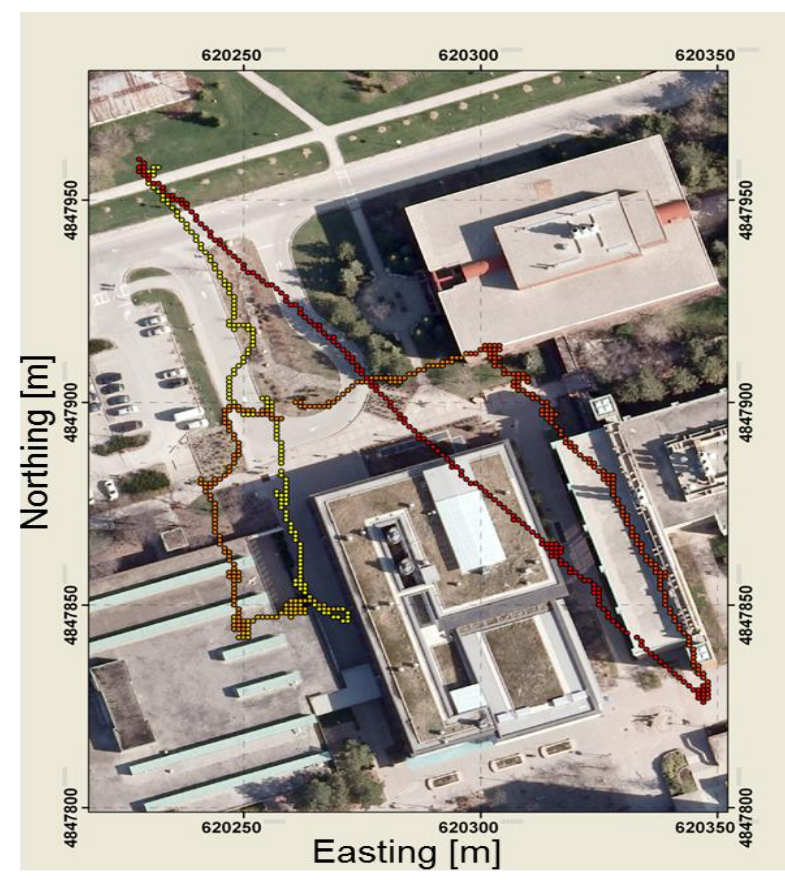

Figure 6. Aeryon Scout's flight path over the Lassonde building
A)
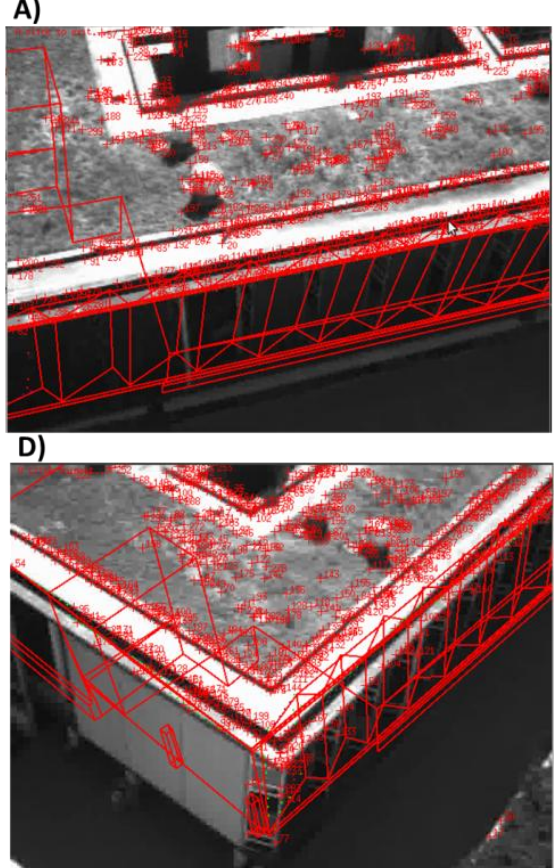

B)
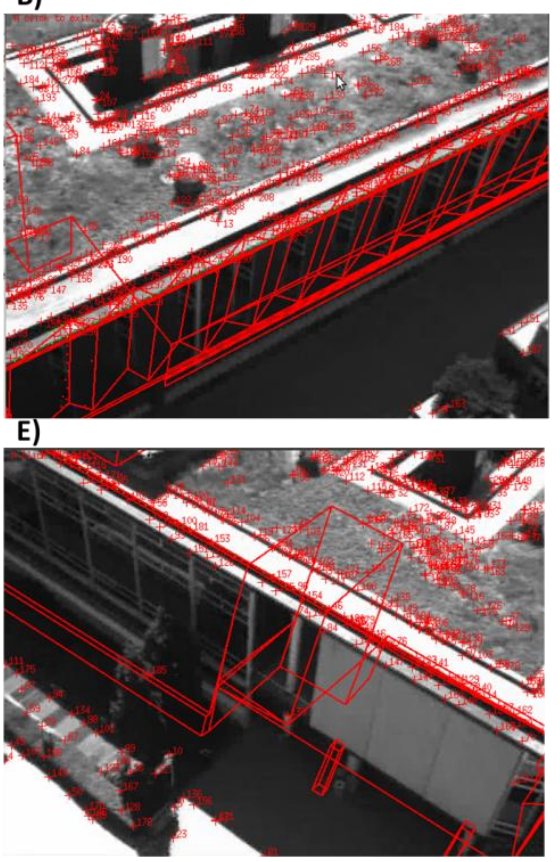

c)

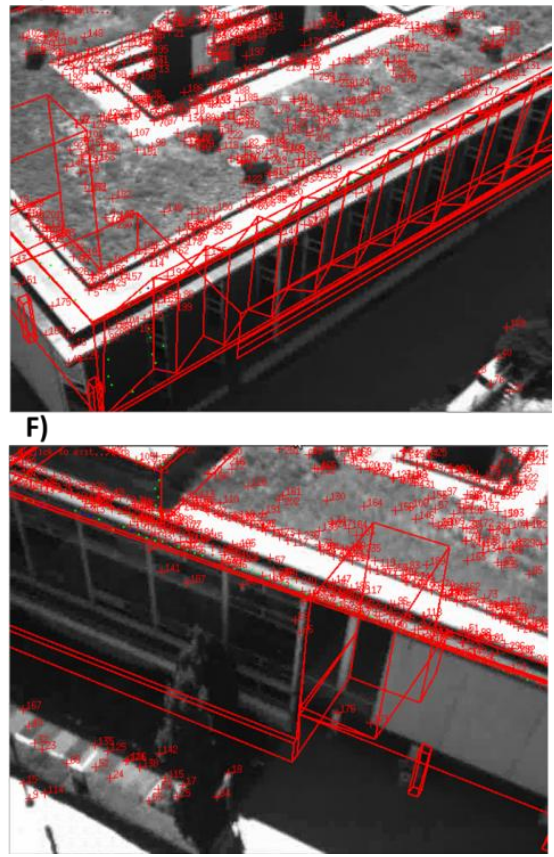

Figure 7. Sample frames demonstrating ViSP's model-based tracker. The West side (A-C) and North side (D-F) of York University's Lassonde Building are being observed, the 3D building model is projected onto the image plane (red lines) using the respective camera intrinsic and extrinsic parameters. Harris corners (red crosses with IDs) are also being tracked. 
A)
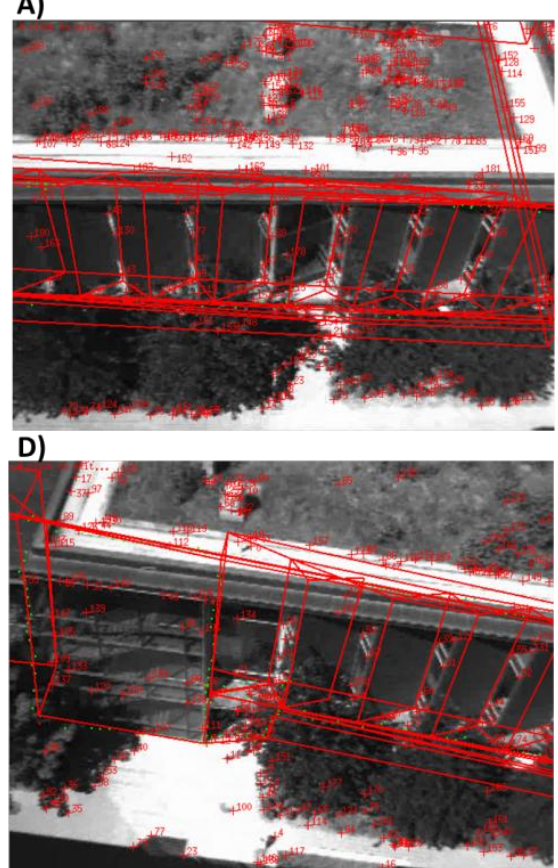

B)

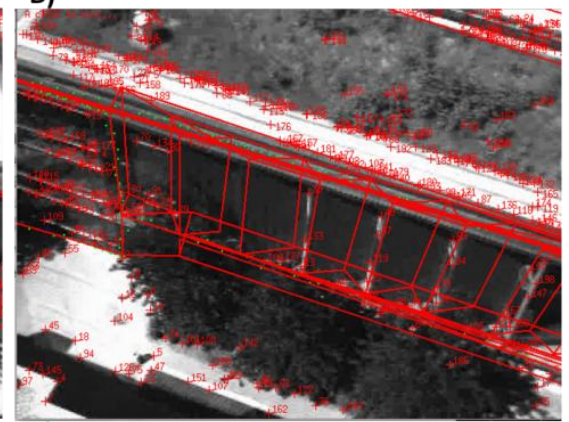

E)

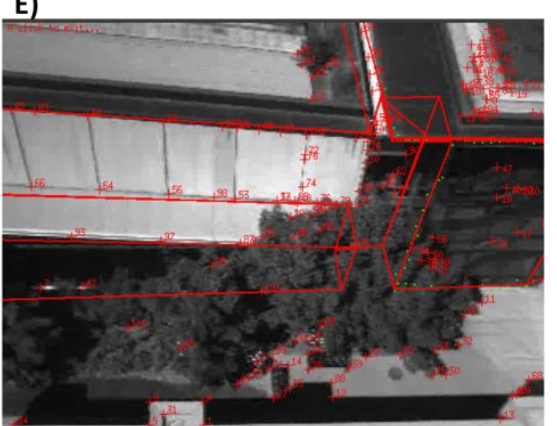

c)

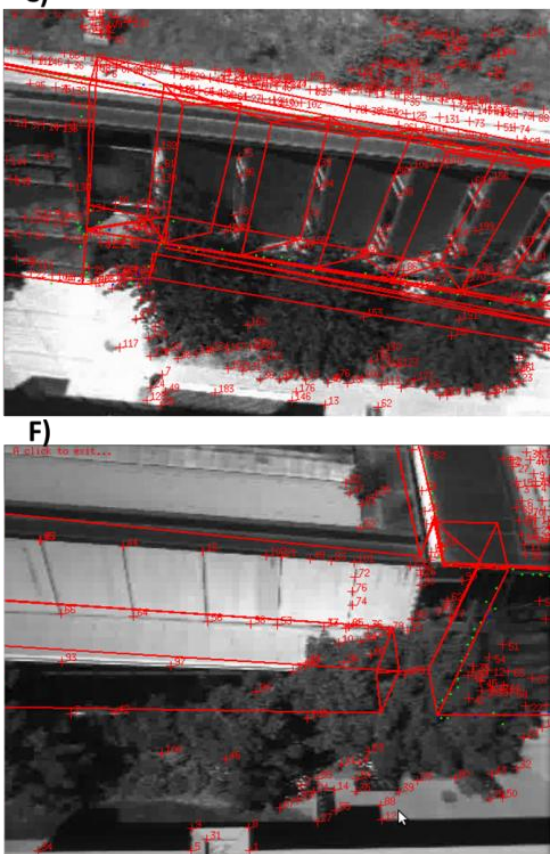

Figure 8. Additional sample frames demonstrating ViSP's model-based tracker. The East side of York University's Lassonde Building is being observed, the 3D building model is projected onto the image plane (red lines) using the respective camera intrinsic and extrinsic parameters. Harris corners (red crosses with IDs) are also being tracked.

The camera's intrinsic parameters were calibrated beforehand and held fixed in the pose estimation process. The image frames were tested at 480p, 720p, and 1080p resolutions. The trials revealed that, at 40 metres above ground level, $480 \mathrm{p}$ video performed the best in terms of processing speed and tracking performance. The tracking performance improved because the noisy edges detected by the Moving Edges Tracker at higher resolutions were removed at lower resolutions, leaving only the stronger edge responses.

1137 frames were processed at 6 frames per second. Figure 7A-F shows several results from the tracking along the West side and North side of the Lassonde Building. The red lines are the projections of the $3 \mathrm{D}$ building model onto the image plane using the respective camera pose. It is evident that, although the tracker does not diverge, there is misalignment between the image and the projected model; this is due to error in the camera pose. Figure 8A-F shows results from ViSP along the East side of the Lassonde Building. Notably, from frame E) to F) in both Figure 7 and 8, ViSP's robust control law decreases the misalignment between the image and model.

Figure 9 shows a comparison between the UAV's trajectory according to the onboard GPS versus ViSP's solution. Figure 10A-C shows the positional coordinates in the $\mathrm{X}, \mathrm{Y}$, and $\mathrm{Z}$ axes, respectively, from the GPS solution and ViSP's solution. Figure 10D shows the coordinate differences between the two solutions, and Table 1 provides the average coordinate differences, along with the standard deviations. These figures show gaps in ViSP's trajectory from when tracking was lost. This was the result from a lack of model features in the camera's field of view, and because of rapid motions due to the unstabilized camera.

The camera was attached the UAV using a pan-tilt mount, so the camera pitched and yawed independently from the UAV. Therefore, the AHRS' roll and pitch axes did not align with the camera frame. However, for both the AHRS' and ViSP's the direction cosine matrix rotation sequences are $\mathrm{ZYX}$, thus their Z-axes are parallel. The camera did not pan throughout the flight, so there was a constant offset between the AHRS and camera yaw angles $\left(60.125^{\circ}\right)$.

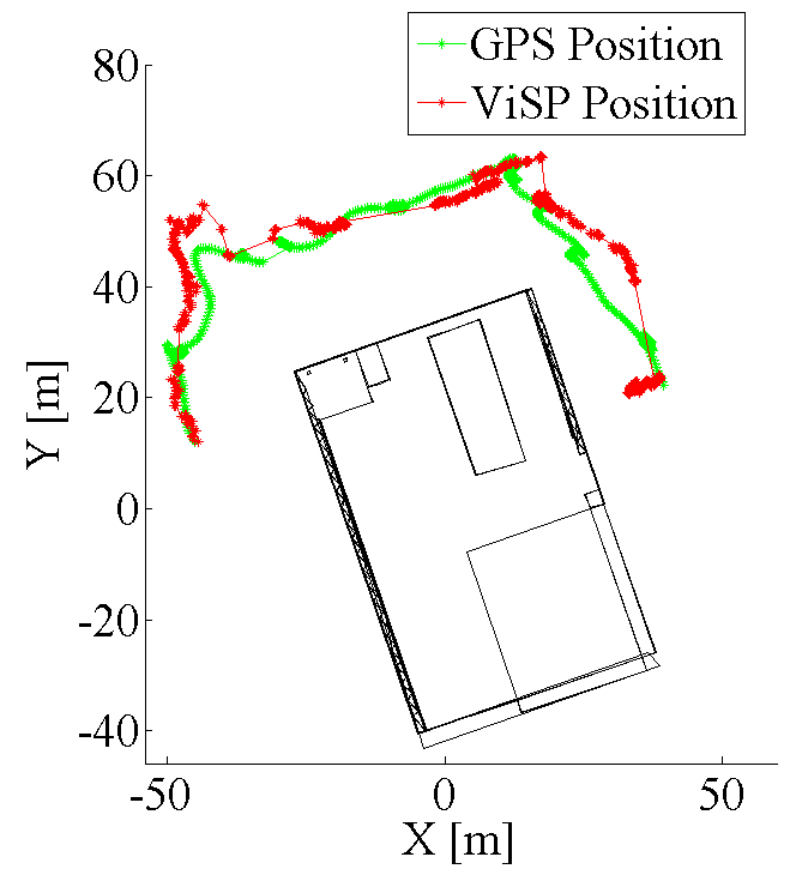

Figure 9. An overhead view of the UAV's trajectory according to the onboard GPS (green) and ViSP solution (red). 

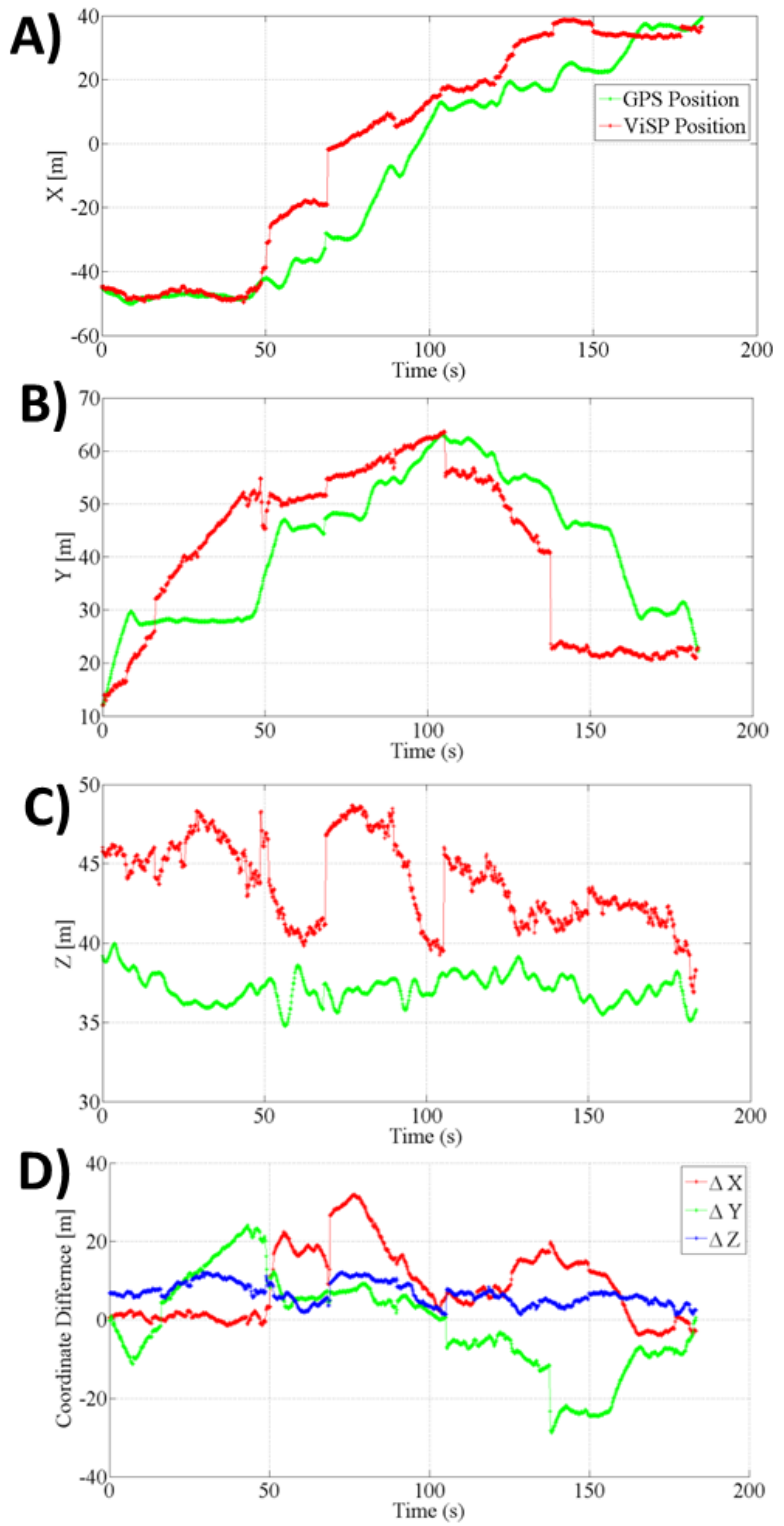

Figure 10. Positional coordinate comparison between the GPS solution and ViSP's solution.

Table 1. Mean and standard deviation for the difference in the UAV's positional coordinates from its onboard GPS and ViSP.

\begin{tabular}{|c|c|c|}
\hline & Mean & $\begin{array}{c}\text { Standard } \\
\text { Deviation }\end{array}$ \\
\hline$\Delta \mathbf{X}[\mathbf{m}]$ & 8.78 & \pm 9.23 \\
\hline$\Delta \mathbf{Y}[\mathbf{m}]$ & -1.19 & \pm 12.16 \\
\hline$\Delta \mathbf{Z}[\mathbf{m}]$ & 6.65 & \pm 2.73 \\
\hline$\Delta \psi\left[{ }^{\circ}\right]$ & 0.15 & \pm 13.87 \\
\hline
\end{tabular}

Figure 11A shows the yaw angles, after removing the offset, and Figure $11 \mathrm{~B}$ shows the difference. It is evident that ViSP's yaw estimate agrees well with the AHRS solution. The jumps in the data again show where tracking was lost and reset. Table 1 provides the mean difference in yaw $(\Delta \psi)$, along with its standard deviation.
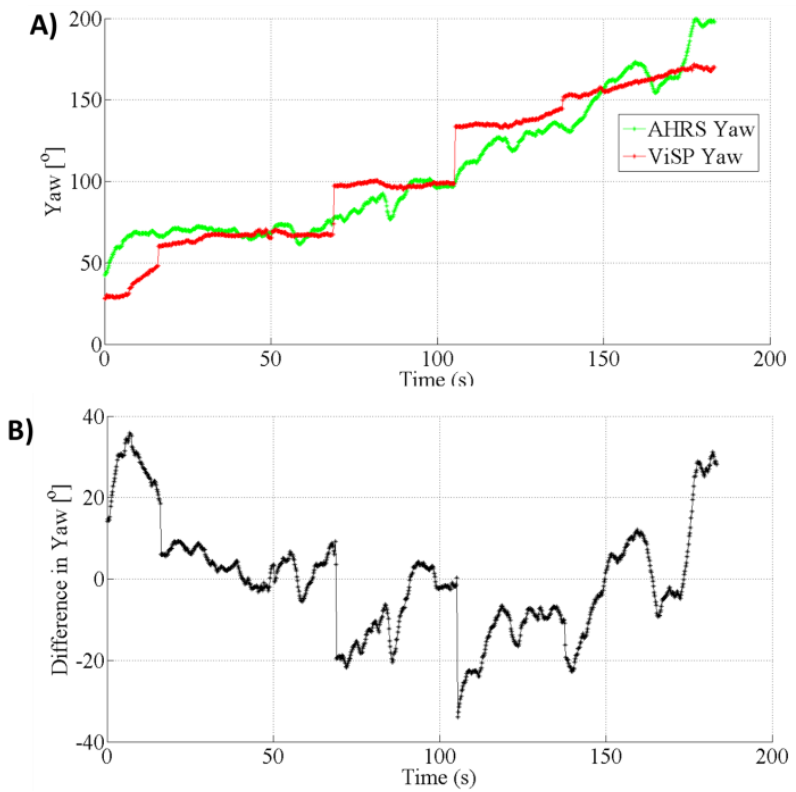

Figure 11. Yaw angle comparison between the GPS solution and ViSP's solution.

The experiments suggested that ViSP can provide only an initial approximation for camera pose. This is because the Moving Edges Tracker is a local matching algorithm. A simultaneous bundle adjustment will globally optimize the camera poses, leading to an increase in the accuracies. Figures 7 and 8 show red points that are labelled with their ID numbers, these are Harris corners being tracked using a KLT tracker (Lucas and Kanade, 1981). They may be used as tie points in the bundle adjustment to further optimize the camera poses and generate a sparse point cloud. Dense matching (Rothermel et al., 2012) could be used to increase the point cloud's density. The Iterative Closest Point (ICP) algorithm (Besl and McKay, 1992) may then be used to refine the alignment between the point cloud and the 3D building model; this should further increase the camera pose accuracies. As both large-scale bundle adjustment, dense matching, and ICP are computationally intensive, they should be done in a post-processing stage.

The real-time camera pose accuracies can be improved by augmenting ViSP's pose estimate with another pose estimation system. For instance, one could integrate ViSP's pose with the positional and orientation data from the UAV's onboard GPS and AHRS, respectively, through a Kalman filter. This would both increase the accuracy of the pose and bridge GPS gaps.

\section{CONCLUSIONS AND FUTURE WORK}

This work explored the application of ViSP in tracking building models from a video sequence collected by a UAV. Our presented experiments demonstrated the data obtainable by the UAV, assessed ViSP's data processing strategies, and evaluated the performance of the tracker. Future work includes implementing a Kalman filter to integrate the UAV's GPS and AHRS data with ViSP's pose estimation system. Then bundle adjustment, dense matching, and ICP will be incorporated to globally optimize the camera poses. 


\section{ACKNOWLEDMENTS}

NSERC's financial support for this research work through a Discovery Grant is much appreciated. We thank Professor James Elder's Human \& Computer Vision Lab in York University's Centre for Vision Research for providing the UAS video data. Special thanks are given to the members of York University's GeoICT Lab Damir Gumerov, Yoonseok Jwa, Brian Kim, and Yu Gao, who contributed to the generation of the York University 3D Virtual Campus Model.

\section{REFERENCES}

Aeryon. 2015. Aeryon Scout Brochure. Retrieved from www.aeryon.com/products/avs/ aeryon-scout.html. (accessed: 14-May-2015).

Armenakis C, and Sohn G. 2009. iCampus: 3D modeling of York University Campus. In Proc. 2009 Conf. of the American Society for Photogrammetry and Remote Sensing, Baltimore, MA, USA.

Besl PJ, and McKay N.D. 1992. A method for registration of 3D shapes. IEEE Trans. on Pattern Analysis and Machine Intelligence (Los Alamitos, CA, USA: IEEE Computer Society) 14(2): 239-256.

Blake A, and Isard M. 1998. Active Contours. Springer Verlag.

Bouthemy P. 1989. A maximum likelihood framework for determining moving edges. IEEE Trans, on Pattern Analysis and Machine Intelligence. 11(5): 499-511.

Corral-Soto ER, Tal R, Wang L, Persad R, Chao L, Chan S, Hou B, Sohn G, and Elder JH. 2012. 3D Town: The Automatic Urban Awareness Project. In Proc. 9th Conf. on Computer and Robot Vision, Toronto, pp. 433-440.

Comport A, Marchand,E, and Chaumette F. 2003. Robust and real-time image-based tracking for markerless augmented reality. Technical Report 4847. INRIA.
Li-Chee Ming J, and Armenakis C. 2014. Feasibility study for pose estimation of small UAS in known 3D environment using geometric hashing. Photogrammetric Engineering \& Remote Sensing, 80(12): 1117-1128

Lucas B.D., and Kanade T. 1981. An iterative image registration technique with an application to stereo vision. In Int. Joint Conf. on Artificial Intelligence, IJCAI'81, pp. 674679.

Marchand E, and Chaumette F. 2005. Feature tracking for visual servoing purposes. Robotics and Autonomous Systems. 52(1): 53-70.

Rothermel M, Wenzel K, Fritsch D, Haala N. 2012. SURE: Photogrammetric surface reconstruction from imagery. Proceedings LC $3 D$ Workshop, Berlin.

Shi Jand Tomasi C. 1994. Good features to track. IEEE Int. Conf. on Computer Vision and Pattern Recognition. pp. 593600 .

Smith P, Reid I, and Davison, A. 2006. Real-time monocular SLAM with straight lines, Proc of the British Machine Vision Conference.

Sundareswaran V, and Behringer R. 1998. Visual servoingbased augmented reality. In IEEE Workshop on Augmented Reality, San Fransisco.

ViSP. 2013. ViSP: Visual servoing platform - Lagadic research platform. Retrieved from http://www.irisa.fr/lagadic/visp/visp.html. (accessed: 22-May2015).

Vincze M. 2001. Robust tracking of ellipses at frame rate. Pattern Recognition. 34(2): 487-498. 